\title{
13 Translational Root Genomics for Crop Improvement
}

\author{
Reyazul Rouf Mir, Mahendar Thudi, Siva K. Chamarthi, \\ L. Krishnamurthy, Pooran M. Gaur, and Rajeev K. Varshney
}

\section{Introduction}

Breeding of crop plants in the 21 st century needs consciousness and awareness of climate change. For instance, both biotic and abiotic stresses need attention of breeders in order to breed crops showing resistance/tolerance to these stresses in a changing climate scenario. In developing countries, drought is the major abiotic stress and is already limiting crop productivity in several species. This tendency is going to worsen the situation in the years to come (Varshney et al. 2011a). Enhancing crop productivity in resource-poor dry land conditions is a formidable challenge. Conserving resources through management practices and engineering plants for superior extraction of these resources coupled with an increased efficiency of resource utilization deserve emphasis. Though resource conservation through management practices are equally important, development of superior resource use efficiency as a seed-based technology always has greater acceptance and adaptability.

Roots, which are underground hidden parts of plants, are considered as first plant organs to be exposed, as well as to respond in stresses. However, among several parts of the plant, roots have received little attention of researchers so far despite their major role in plant-soil interactions (Sheshshayee et al. 2011). Thus, they seem to hold the key for the next plant breeding revolution, leading to improved crop productivity in environmentally challenged situations. Recent advancements in plant genomics are certainly of help in crop improvement efforts (Varshney et al. 2005) and genomics-assisted breeding applications such as marker-assisted selection (MAS) have been already used in developing superior cultivars in several crop species (Varshney et al. 2006, 2010; Gupta et al. 2010). The field of root genomics is an exciting and promising field of research and some technical advancements in plant-omics are believed to generate some useful data for pursuing translational research. Whole genome approaches such as microarrays or next-generation sequencing (NGS)-based transcript profiling or functional genomics approaches may provide some useful information about the type and nature of structural and functional genes involved in various aspects of root growth, development, and water and nutrient uptake. In addition, on the basis of whole genome sequence available for several crop species (for references, see Jackson et al. 2011; Morrell et al. 2012) or gene information available in model plant species, use of comparative genomics or bioinformatics approaches may provide interesting candidate genes. Once the details of these genes are known, some other approaches like overexpression, TILLLING (Targeting-Induced Local Lesions IN Genomes; Varshney et al. 2011b), qRT-PCR, gene-knockout mutant analysis may be used to further validate their utility in root trait improvement.

Root Genomics and Soil Interactions, First Edition. Edited by Martin Crespi.

(C) 2013 John Wiley \& Sons, Inc. Published 2013 by John Wiley \& Sons, Inc. 
In this article, we highlight the importance of root genomics research in the context of drought stress and discuss recent developments in genetic and molecular dissection of root traits. A special emphasis has been given on translational root genomics for developing superior varieties through molecular breeding for root traits.

\section{Root Research for Crop Improvement}

Studying roots in higher plants assumes importance because they provide firm anchorage of the plant in their soil substratum and helps in absorption and effective supply of water and nutrients to the shoot. These are the most important roles of the root system (Varshney et al. 2011b). In addition, roots assume importance for plants by producing a number of growth hormones, including cytokinins and ABA. From an ecological point of view, roots play some role in weathering of rocks, thus leading to the formation of soil. Sometimes, tiny roots spread out by growing horizontally and form a thick mat-like network; they may prevent soil erosion and through their symbiotic association, roots with rhizobia, mycorrhiza, and other organisms can fix atmospheric nitrogen or enrich mineral soil content. However, keeping in view the current scenario of importance of drought in view of changing climate, the water mining capacity of roots from deeper soil profiles is considered as one of the important adaptive strategies evolved by plants to survive water-scarce conditions. With an objective of paying more attention on root research at genetic, physiological, and molecular level, a Plant Root Genomics Consortium was developed by University of Missouri in collaboration of other partners from USA (http://rootgenomics.rnet.missouri.edu/prgc/index.html). The primary goal of this consortium is to develop understanding of molecular mechanisms used by plant roots to get water and nutrition from soil and to find out the possible role of roots in adaptation to drought conditions and further transfer this knowledge for crop improvement through various breeding and biotechnological approaches. Discussions are currently underway to extend this consortium into an International Root Genomics Consortium.

The role of roots is very significant to enhance drought tolerance. In simpler terms, drought tolerance is the ability of a plant to avoid or tolerate stress at organism level (Levitt 1972; Blum 2005). Drought is often regarded as a major threat to ecosystems, as water stress limits crop yield more than all other biotic and abiotic factors combined (Lambers et al. 2008). In these situations, the ability of the plants to explore water resource by reaching roots deep in the soil and extracting water has great relevance in maintaining water relation as well as carbon assimilation. Therefore, genotypes with long deep-roots have been found more tolerant to drought (Li et al. 2005a; Kashiwagi et al. 2005, 2006; Reynolds and Tuberosa 2008). In addition, some other related traits have been suggested to help plants to perform better in water-limited conditions. For instance, low leaf conductance under nonlimited water conditions during the vegetative stage, higher fraction of transpirable soil water (FTSW) thresholds that reduce transpiration, thus avoiding rapid soil water depletion, and low leaf expansion rate when soil moisture is still nonlimiting for plant growth have been suggested in chickpea (Zaman-Allah et al. 2011).

\section{Genetic Dissection of Root Traits}

\section{Quantitative Trait Locus (QTL) Discovery for Root Traits}

In simple terms, a QTL is a segment of DNA that affects a quantitative trait or the region within genome that contains genes associated with a particular quantitative trait. The plant root system is 
considered highly dynamic and responds to changes in environmental parameters, including stresses such as drought, nutrient deficiencies, water logging, and salinity. However, despite their essential role in plant growth, adaptation, and mineral nutrient acquisition, the root system has remained unexplored, owing to the difficulty in screening techniques until recently. Once the considerable understanding of root growth and development, both at the whole plant level and at the molecular level, is achieved, the next step is to devise strategies for identification of important genes/QTLs associated with various root traits followed by their validation and subsequently introgression into crops through molecular breeding approaches (Varshney et al. 2011b). In terms of genetic control, root traits are believed to be complex controlled by a number of genes/QTLs. Therefore, understanding the genetic control of root development and functions of component root traits is considered inherently important for breeding improved cultivars for root traits that are well adapted to variable climates. For QTL mapping studies, generating large-scale and precise phenotyping data on mapping populations is very critical. In recent years, significant progress has been made toward improving phenotyping capabilities (Manschadi et al. 2008; Gregory et al. 2009; Hund et al 2009; Nagel et al. 2009; Yazdanbakhsh and Fisahn 2009; Chen et al. 2011) as well as marker genotyping capacities (see Mir et al., in press). As a result, marker-trait association (MTA) studies are gaining importance to gain new insights into the genetic control of root system architecture (see de Dorlodot et al. 2007; Courtois et al. 2009; Hochholdinger and Tuberosa 2009; Coudert et al. 2010; Kell 2011; Gowda et al. 2011; Tuberosa et al. 2011).

In general, there are two important approaches for the study of MTA: (i) QTL interval mapping commonly called linkage mapping/linkage analysis-based QTL mapping and (ii) linkage disequilibrium-based association mapping (Figure 13.1). Both approaches have their own advantages and disadvantages and have been already used for discovering QTLs/genes for a variety of traits in all important crop plants (see Myles et al. 2009; Rafalski 2010). The various steps involved in both approaches have been discussed elsewhere (see Varshney et al. 2011b; Chamarthi et al. 2011; Figure 13.1). By using one of these two approaches, QTLs for root traits have been identified in about 15 plant species (Kalliokoski et al. 2008). However, rice, maize, and wheat among cereal crops and soybean, common bean and chickpea among legumes have dominated root trait QTL studies. Some of these important QTL studies have been summarized in Table 13.1.

Rice is probably the crop species in which a maximum number of QTL studies for root traits have been targeted (see Courtois et al. 2009; Priya et al. 2009). More than 900 QTLs related to root traits such as maximum length, number, thickness, volume, and their effects on yield under varying moisture regimes have been studied in rice (Kamoshita et al. 2002a; MacMillan et al. 2006; Steele et al. 2006, 2007; Yue et al. 2006). Since precise phenotyping is considered crucial in the study of MTA for root traits, a range of phenotyping platforms and methods including green house (Komoshita et al. 2002b), hydroponic cultures (Obara et al. 2010), basket method (Uga et al. 2011), poly vinyl chloride (PVC) cylinders (Qu et al. 2008), and field conditions (Ikeda et al. 2007; Yue et al. 2008) have been used. In terms of genetic localization, QTLs for maximum root length have been identified almost on all 12 chromosomes (Hemamalini et al. 2000; Zhang et al. 2001; Kamoshita et al. 2002a, 2002b; Courtois et al. 2003; Horii et al. 2006; MacMillan et al. 2006; Yue et al. 2006). A major QTL for root length (qRL6.1) based on phenotyping of seedlings grown under hydroponic conditions has been identified on chromosome 6 (Obara et al. 2010) and delimited to a $337 \mathrm{~kb}$ region of the Nipponbare genome. Another major QTL controlling RDR (for ratio of deep rooting), which explains $66.6 \%$ total phenotypic variation (PV) was detected on chromosome 9 by using RIL populations derived from a cross IR64 × Kinandang Patong (Uga et al. 2011).

In the case of maize, QTLs for various root traits have been identified based on phenotyping using a range of soil moisture regimes (Tuberosa et al. 2002a, 2003; Landi et al. 2007). QTLs of root pulling resistance at flowering time were identified based on phenotyping in field conditions (Giuliani et al. 


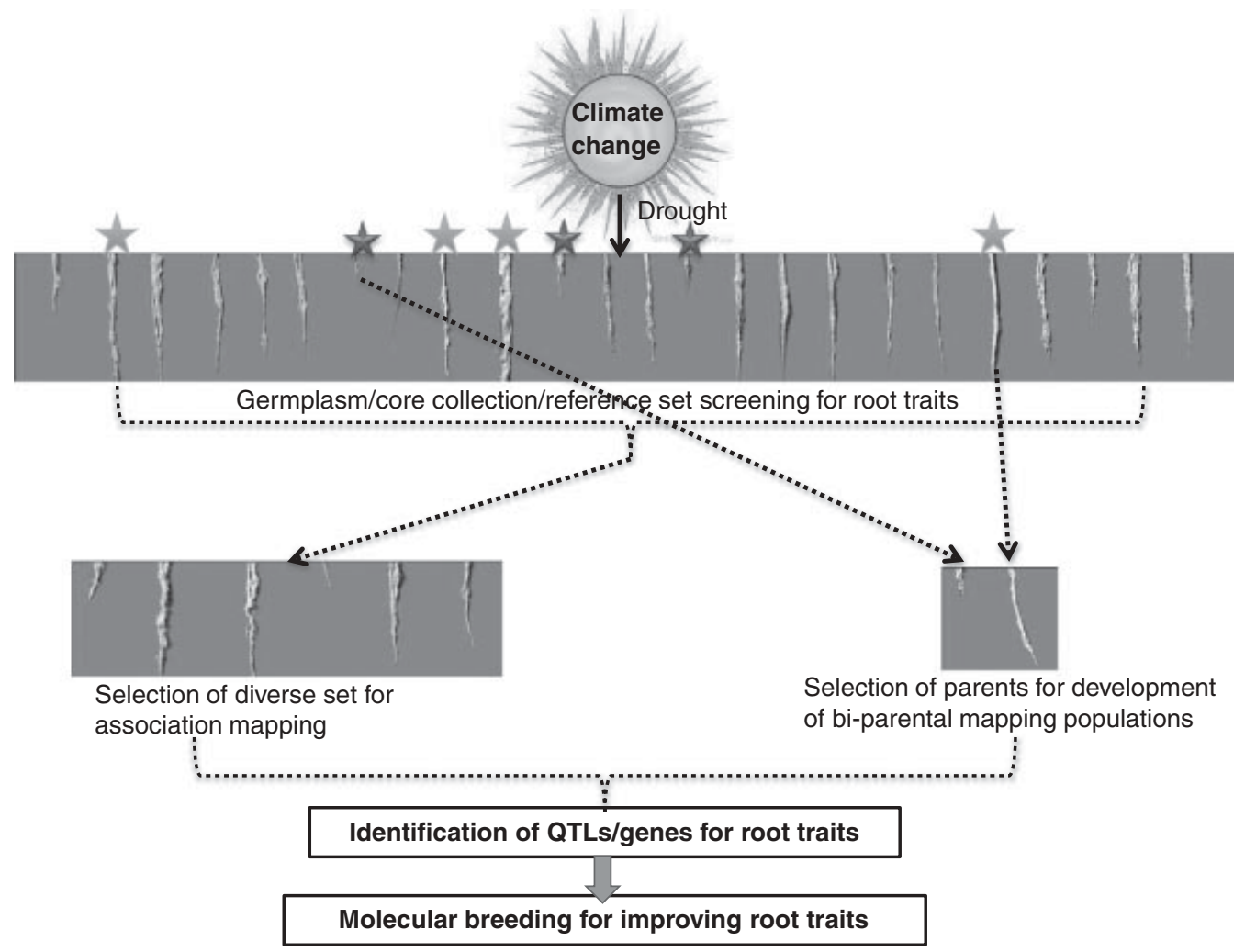

Figure 13.1 A schematic figure showing discovery and translation of root trait QTLs for crop improvement. The figure shows screening of germplasm under natural conditions for root traits. While a diverse set of germplasm showing a variation of root traits is selected and used for association mapping, contrasting lines comprising of higher root, and lower root biomass are selected for developing biparental mapping populations and linkage mapping-based QTL analysis. Once major QTLs are identified, molecular breeding for these QTLs can lead to development of superior lines for improved root traits. Red stars indicated lines with short roots (mostly drought susceptible), while green lines indicated lines with long roots (mostly drought tolerant). (For a color version of this figure, see the color plate section.)

2000). Recently, Ruta et al. (2010) identified 13 QTLs for six seedling traits: elongation rates of axile roots, the rate constant of lateral root elongation (kLat), the final respective lengths LAx and LLat, and the ratios kLat/ERAx and LLat/LAx. In this study, QTLs for the elongation rates of axile roots responded more clearly to water stress compared to root length. In addition, many QTLs for root traits and their responses to drought and phosphorus deficiency have been reported in maize (Lebreton et al. 1995; Guingo et al. 1998; Landi et al. 2002; Tuberosa et al. 2002b; Hund et al. 2004; Mano et al. 2005; Zhu et al. 2005a, 2005b, 2006). However, QTL analysis for root traits in response to low $\mathrm{N}$ stress is not well studied.

In the case of wheat, Ma et al. (2005) found a QTL for root-growth rate under Al treatment. QTLs of root traits (primary/lateral root length and number, root dry matter) under control conditions and during nitrogen deficiency were also identified (Laperche et al. 2006). Several studies identified QTLs for early root growth in wheat (An et al. 2006; Laperche et al. 2006; Li et al. 2007; Sanguineti et al. 2007; Sharma et al. 2011). In a recent study, a total of 15 QTLs, including 6 additive and 9 


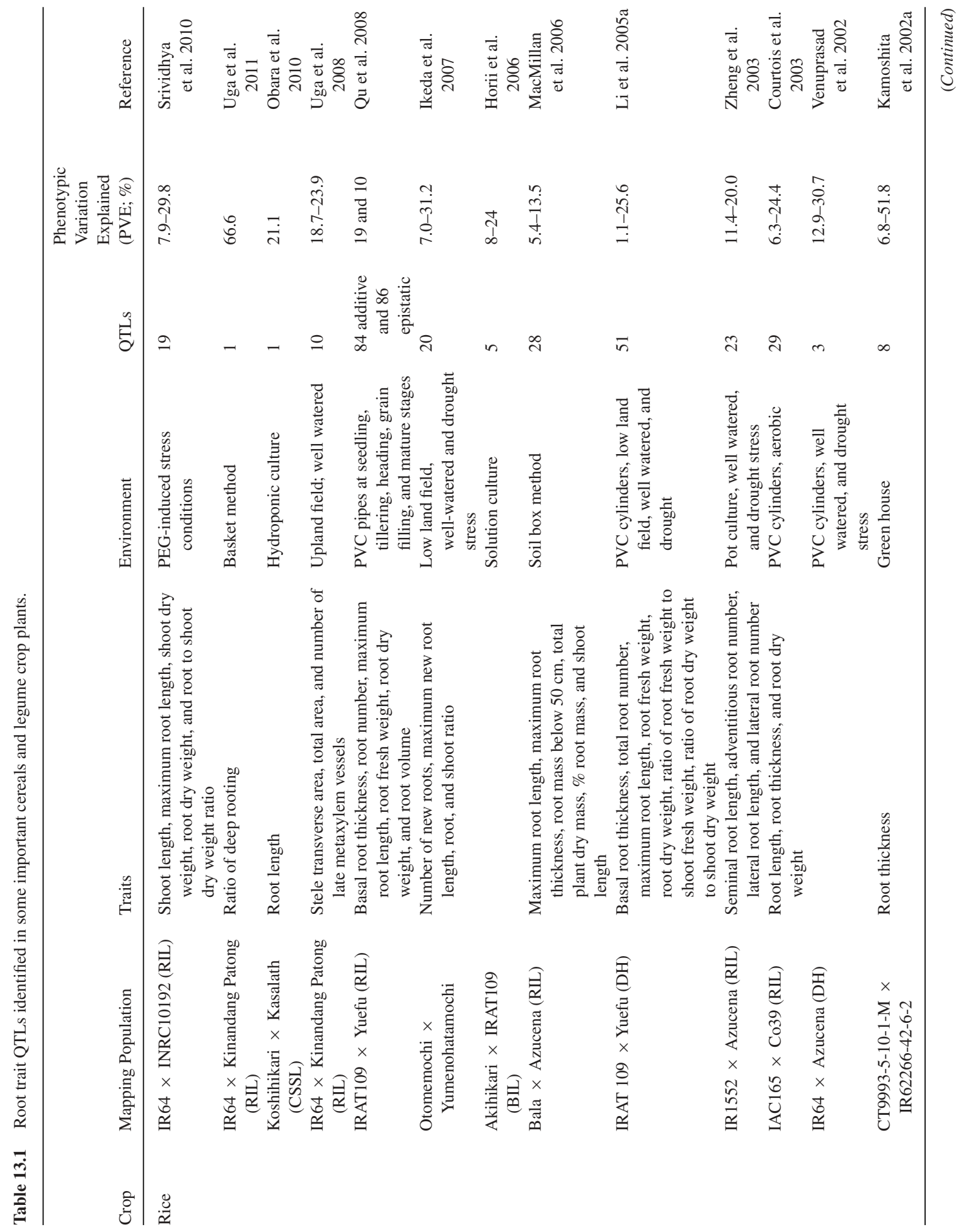




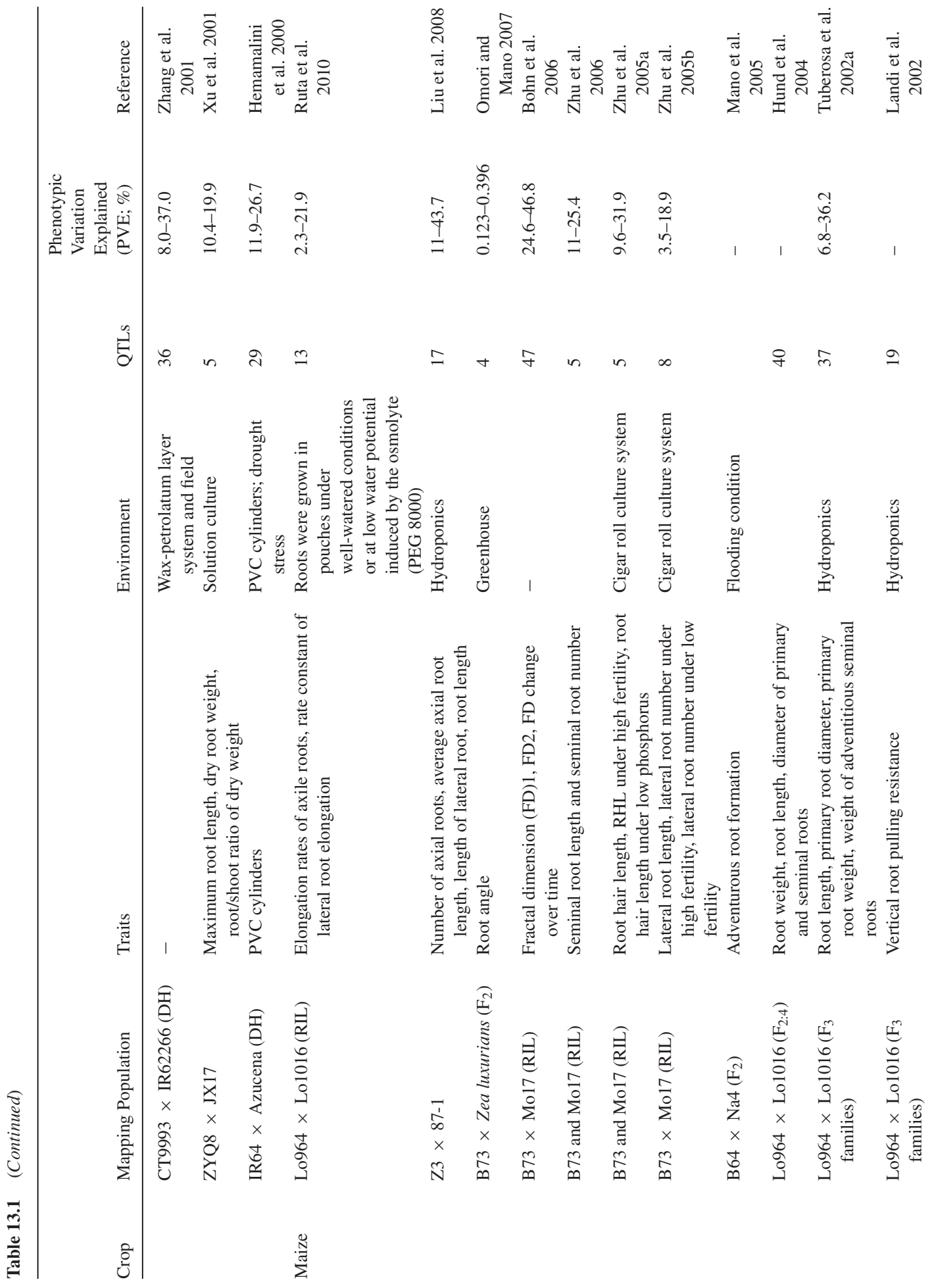




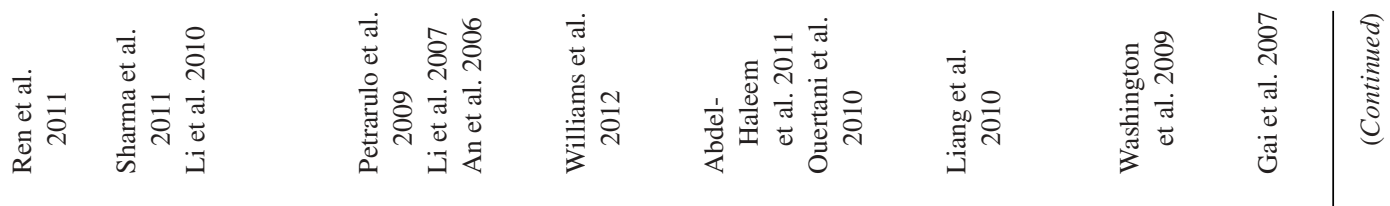

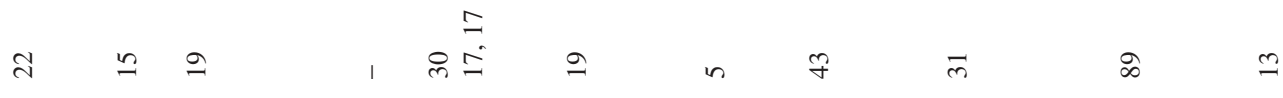

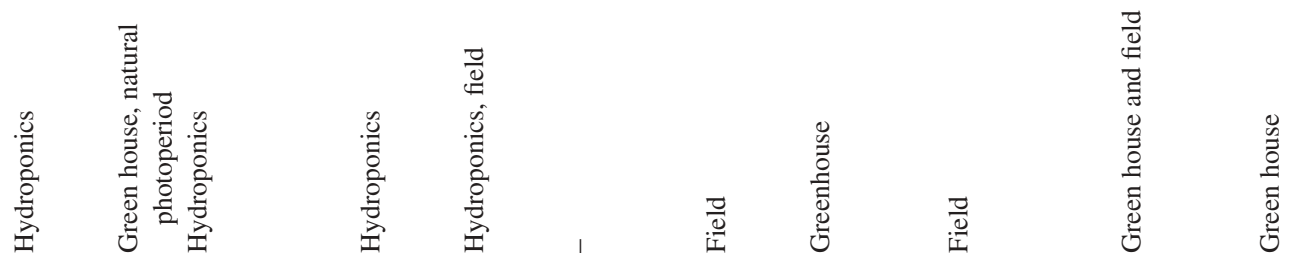

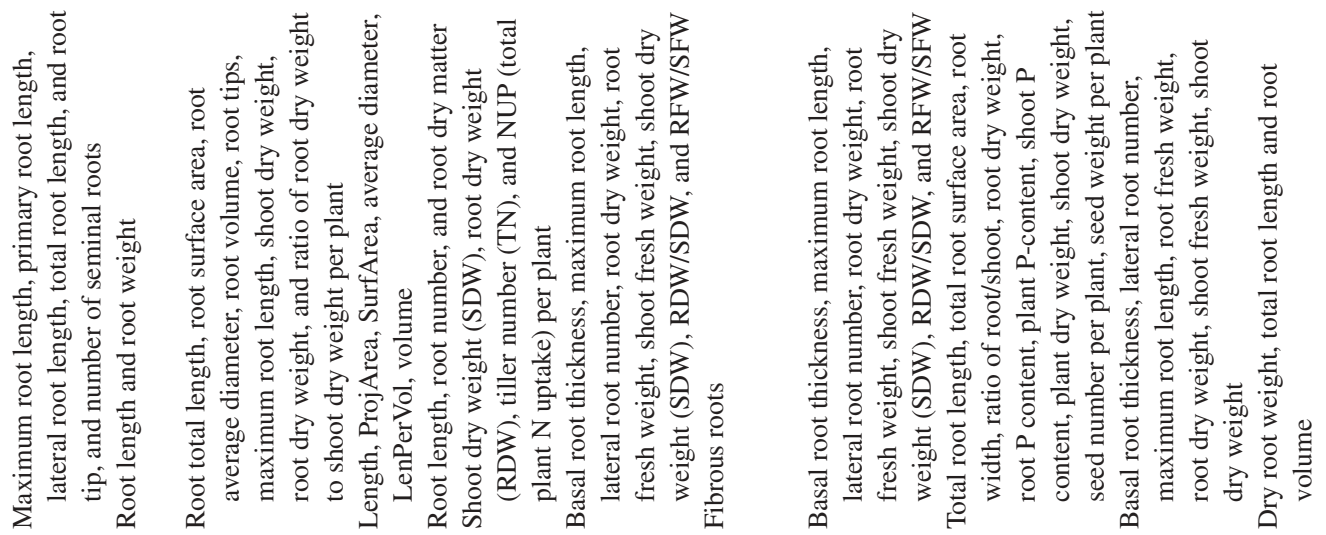

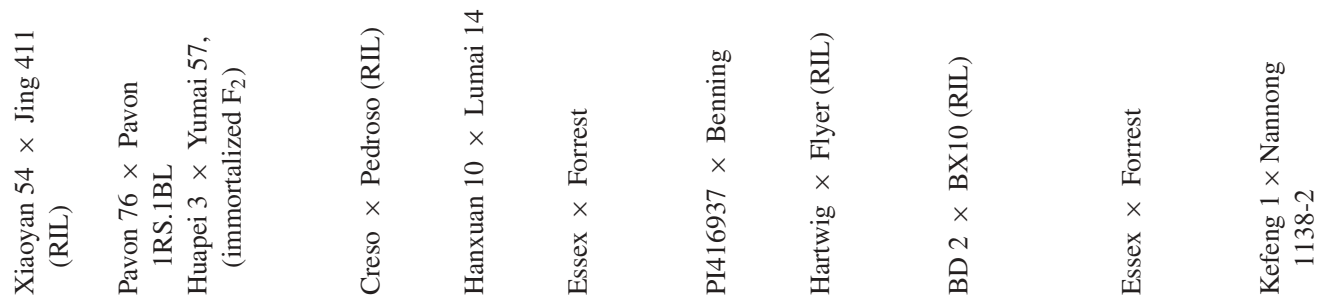




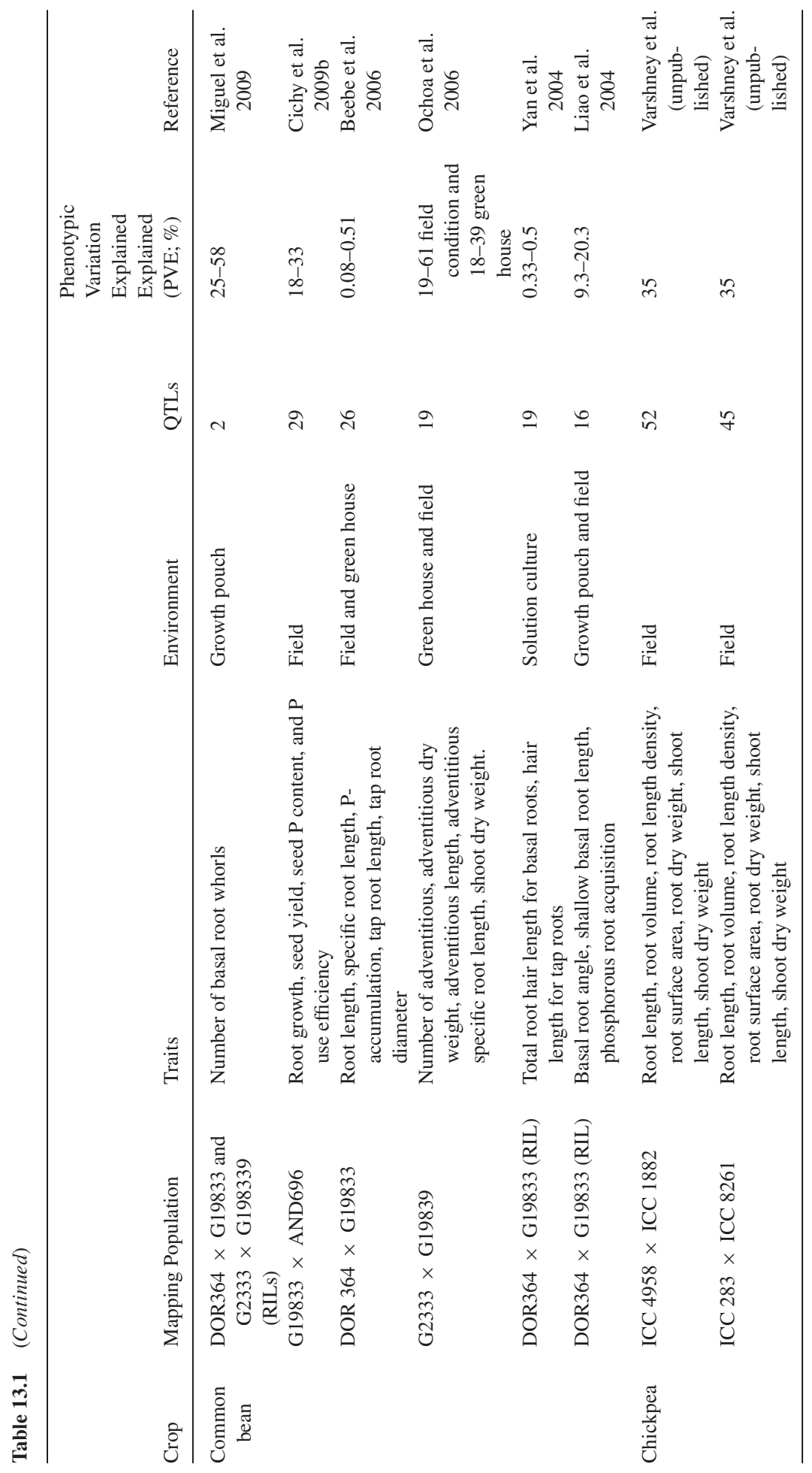


epistatic QTLs have been detected for different traits of root length and root weight on 1RS segment using a high-resolution chromosome arm-specific mapping population (Sharma et al. 2011). In the case of sorghum, nodal root angle (qRA) that influences vertical and horizontal root distribution in the soil profile is considered one of the most important traits that may provide new opportunities for improving drought adaptation mechanisms. Mace et al. (2012) have reported four QTLs for qRA in addition to three QTLs for root dry weight: two for shoot dry weight and three for plant leaf area. Further, qRA QTL explained $58.2 \%$ of the phenotypic variance was also validated across a range of diverse inbred lines.

Among legumes, QTLs for root fresh weight, maximum root length, basal root thickness, lateral root number, root dry weight, total root surface area, root width, ratio of root/shoot, root dry weight, root $\mathrm{P}$ content, plant $\mathrm{P}$ content, and shoot $\mathrm{P}$ content have been identified in soybean under field conditions (Liang et al. 2010; Abdel-Haleem et al. 2011) as well as under greenhouse conditions (Gai et al. 2007; Washington et al. 2009). On the other hand, a large number of QTLs for P efficiency have been identified in soybean ( $\mathrm{Li}$ et al. 2005b; Zhang et al. 2009). In the case of common bean, a large number of QTLs for root morphology and physiology as related to P nutrition have been reported (Beebe et al. 2006; Li et al. 2007; Chen et al. 2009; Cichy et al. 2009a; Li et al. 2009). More interestingly, QTLs for basal root growth and development were shown tightly linked with the QTLs for P uptake efficiency under field conditions (Beebe et al. 2006). In the case of chickpea, based on phenotyping of root traits under PVC cylinder and SSR genotyping on two mapping populations (ICC $4958 \times$ ICC 1882 and ICC $283 \times$ ICC 8261), a genomic region, harboring QTLs for various drought tolerance related root traits, has been identified on linkage group 4, which explained more than $35 \%$ phenotypic variation (unpublished data).

\section{Meta-QTL Analysis for Root Traits}

As different QTL studies employ different mapping populations like recombinant inbred lines (RILs), doubled haploids (DHs), backcross introgression lines, and near isogenic lines (NILs) that segregate for different root traits, it is difficult to exploit the varietal difference for improved root traits by MAS and for identification of concerned alleles. In this context, meta-analysis of QTLs is considered one of the best approaches that is conducted when hundreds of QTLs based on large number of studies involving a number of mapping populations are available in the published literature. This approach is a useful reductionist approach to bring down the number of genuine and real QTLs for their efficient use in MAS. Meta-analysis of QTLs provides narrow confidence intervals for meta-QTLs (MQTLs), permitting easier positional candidate gene identification. Metaanalysis of QTLs is usually applied to multiple mapping populations, but can be applied to one also (Khowaja et al. 2009).

For comparing root traits across experiments and developmental stages, the International Society of Root Research (ISRR, www.rootresearch.org) proposed a general nomenclature for roots (Zobel and Weisel 2010) to compare root types in different crop species. Recently, a database "QlicRice" was developed by Smita et al. (2011), which serves as a web interface and platform for data mining of abiotic stress responsive QTLs and their corresponding sequenced gene loci, which help researchers to retrieve association among different agronomic traits. Meta-analysis of drought-related QTLs in the Bala $\times$ Azucena mapping population of rice from 13 experiments and 25 independent screens resulted in three meta-QTLs within a space of $35 \mathrm{cM}$ on chromosome 9 (Khowaja et al. 2009). Swamy et al. (2011) identified that the MQTL for grain yield under drought coincided with at least one of the MQTL identified for root and leaf morphology traits under drought in earlier reports. 
A rational database "Rootbrowse" has also been constructed using QTLs, markers, and genome sequence information (Priya et al. 2009).

In the case of maize, MQTL analysis based on 15 independent QTL studies of 9 mapping populations resulted in the identification of seven MQTL for root traits that are colocalized with grain yield and other drought responsive traits in the field. Furthermore, on the basis of MQTL analysis, Landi et al. (2010) inferred that one single locus, root-yield-1.06, has a major constitutive effect on root traits, plant vigor and productivity across water regimes, genetic backgrounds, and inbreeding levels.

\section{Molecular Dissection of Root Traits}

\section{Candidate Genes Based on Genome Sequence Information}

As evident from Table 13.1, the root trait QTLs have been studied extensively in rice than any other crop. It is also known that the rice root system is complex, consisting of seminal, adventitious, and lateral roots. The availability of the rice genome sequence has opened some new avenues to look at the large number of QTLs already identified to control root development and to identify candidate genes in those QTL regions. In this context, a majority of root QTLs identified in rice were integrated computationally into the japonica genome and it was observed that a maximum number of QTLs for root development is present on chromosome 1 and fewer QTLs on some other chromosomes (Ulaganathan et al. 2007; http://www.kulab.org/research1.htm). As expected, several QTLs reported in different studies utilizing different mapping populations have been found to possess QTLs in the same genomic regions, thus showing that many QTL regions are common across different mapping populations. Some of these genomic regions possessing several overlapping QTLs could also be resolved into multiple QTLs. More interestingly, the putative candidate genes governing root development in these QTLs regions were extracted and their putative functions were computationally analyzed. This led to identification of small number of genes (200-300 genes) in some of the QTL regions, while a large number of genes (600-800 genes) were present in larger QTLs/genomic regions. In summary, computational analysis of the genes revealed and confirmed the polygenic and complex nature of root development in rice.

These genes identified in genomic regions for root development mostly belongs to categories of (a) transcription factors, (b) auxin metabolism and transport genes, (c) auxin-responsive genes, (d) auxin-related proteasome pathway genes, (e) environmental sensors, and (f) biotic and abiotic stress tolerance genes (http://www.kulab.org/research1.htm). However, it will be essential to validate these genes using functional genomics approaches like quantitative RT-PCR or reverse genetic approaches like knockout mutant analysis and TILLING. Nevertheless, availability of the genome sequence in several crop species like maize (Schnable et al. 2009), soybean (Schmutz et al. 2010), sorghum (Paterson et al. 2009), pigeonpea (Varshney et al. 2012), and others offer the possibility to identify the candidate genes underlying the QTL regions identified in these crop species.

\section{QTL Cloning for Root Traits}

Most of the QTL mapping studies involves crossing of varieties with contrasting root characteristics, development of mapping populations, and identification of number of QTLs on different chromosomes. Since most of these experiments involve the use of different sets of markers and localization 
of QTLs on long genomic regions, thus making it difficult to exploit the varietal difference for improved drought tolerance by MAS and for identification of the concerned alleles. Therefore, one would like to move close to the target QTL or even use perfect functional markers for the introgression of respective QTL/gene for a root trait through molecular breeding. Therefore, efforts may be made to clone all the major and important QTL for a root trait. The positional cloning of a major QTL requires (i) large mapping population (>2000 plants) obtained after crossing two NILs for the target QTL, (ii) identifying the genomic region covering the QTL region, and (iii) validating of effect of candidate gene(s) on phenotype (see Salvi and Tuberosa 2005; Tuberosa and Salvi 2006).

Several reports are available on mapping of QTLs for different traits (see Salvi and Tuberosa 2007); however, only few reports are available where efforts have been made to clone the root trait QTLs. The major obstacle, in this context, is precise phenotyping for root traits in the thousands of plants. Nevertheless, some efforts have been made to clone the QTLs for root traits. For instance in maize two major QTLs on chromosome bins 1.06 and 2.04 (root-ABAl) affecting root architecture and a number of agronomic traits, including grain yield, have been targeted (Tuberosa and Salvi 2007). Similarly, efforts are underway for fine mapping/cloning of "root-ABAl" QTL that is responsible for root architecture and leaf ABA concentration in pearl millet (Kholova et al. 2010a, 2010b). In future, newer genomics approaches like association mapping (Rafalski 2010) and next generation sequencing (Varshney et al. 2009) are expected to facilitate cloning of QTLs for root traits.

\section{Molecular Breeding for Root Traits}

MTA studies identify important genomic regions, explaining a significant proportion of PV for root traits. Once major QTLs are identified, molecular markers associated with these QTLs need to be validated on a range of germplasm to select the candidate QTLs as well as the elite cultivars for introgression of the QTL using marker-assisted backcross (MABC) approach. This approach has been successfully used in several crops for improving different traits (Gupta et al. 2010; Varshney et al. 2010; Chamarthi et al. 2011; Kulwal et al. 2011). However, the power and efficiency of this approach has been limited, especially for root traits. For instance, Steele et al. $(2006,2007)$ demonstrated the power of MABC by releasing the first novel upland rice variety Birsa Vikas Dhan 111 (PY 84) through molecular breeding in the Jharkhand state of India. The variety was bred through MABC involving introgression of three genomic regions carrying root growth QTL on chromosomes 2 (root length), 9 (root thickness), and 11 (root penetration) from the donor Philippines variety Azucena into the recurrent parent Kalinga III. The target QTLs were first identified by Adam Price (now at Aberdeen University, UK) and Birgitte Courtois (CIRAD, France/IRRI, Philippines; http://www.cazs.bangor.ac.uk/ccstudio/WhatsNew/cazsWhatsNew2.php?ID=14; Cairns et al. 2009; Courtois et al. 2009; Norton and Price 2009). The variety developed in a collaborative partnership between Centre for Arid Zone Studies (CAZS) Natural Resources, Bangor University, UK; Gramin Vikas Trust, Ranchi, Jharkhand, India; and Birsa Agricultural University, Ranchi, Jharkhand, India, showed improved root growth, thus leading to improved performance under drought conditions. Similarly, Shen et al. (2001) also showed the effectiveness of QTL-based transfer of root traits, by involving the transfer of Azucena allele at four QTL alleles for deeper roots (on chromosomes 1, 2, 7, and 9) from selected DH lines into IR64. Thus, they demonstrated that NILs with an improved root system permit testing the importance of root depth for water-limited environments. In the case of chickpea also, a hotspot harboring several QTLs for drought tolerance-related traits (with $>30 \% \mathrm{PV}$ ) is being introgressed into a leading chickpea variety JG11 through MABC approach 
(unpublished results). Using both foreground and background selection with SSR/AFLP markers, $\mathrm{BC}_{3} \mathrm{~F}_{4}$ progenies have been derived and are being evaluated for their agronomic performance under rainfed and irrigated conditions in India, Kenya, Ethiopia, and Tanzania. These are some of the nice examples of translational genomic research for crop improvement.

\section{Summary and Outlook}

Roots are considered primary targets in view of current scenario of importance of drought in changing climate. Therefore, it is important to understand genetic and molecular mechanisms involved in conferring drought tolerance through root traits. While a linkage mapping-based approach has been used extensively to identify QTLs for a range of root traits in some crop species, association mapping has also started to be used in some cases for identification of QTLs. Some efforts have also been made toward cloning of QTLs for roots traits in species like maize and pearl millet. In terms of translation, a few reports have become available on molecular breeding through MABC and superior varieties have been developed. MABC approach for translating root genomics is possible only in the case where QTLs contribute to higher PV. In the cases where root traits are controlled by many and smalleffect QTLs, the MARS approach that involves intermating selected individuals in each selection cycle (Eathington et al. 2007; Ribaut and Ragot 2007; Bernardo 2008) seems to be a better approach. Genome-wide selection or genomic selection approach is the other approach that involves selection of genotypes based on genomic estimated breeding values, estimated using genome-wide marker profile data for making the crosses (Bernardo and Yu 2007). Availability of genome sequences, NGS technologies, high-throughput genotyping, as well as phenotyping facilities are expected to accelerate root genomics research, especially for translation to crop improvement.

\section{Acknowledgments}

Authors are thankful to CGIAR Generation Challenge Programme (GCP) for providing ThemeLeader Discretionary grant and Tropical Legume I (TLI) project and Department of Biotechnology (DBT) of Government of India for Centre of Excellence $(\mathrm{CoE})$ grant that has made it possible to write this article.

\section{References}

Abdel-Haleem, H. et al. (2011) Identification of QTLs for increased fibrous roots in soybean. Theoretical and Applied Genetics, $122,935-946$.

An, D.G. et al. (2006) Mapping QTLs for nitrogen uptake in relation to the early growth of wheat (Triticum aestivum L.). Plant Soil 284, 73-84.

Beebe, S.E. et al. (2006) Quantitative trait loci for root architecture traits correlated with phosphorus acquisition in common bean. Crop Science, 46, 413-423.

Bernardo, R. (2008) Molecular markers and selection for complex traits in plants: learning from the last 20 years. Crop Science, 48, 1649-1664.

Bernardo, R. and Yu, J. (2007) Prospects for genome wide selection for quantitative traits in maize. Crop Science, 47, $1082-1090$.

Blum, A. (2005) Drought resistance, water-use efficiency and yield potential—are they compatible, dissonant or mutually exclusive? Australian Journal of Agricultural Research 56, 1159-1168.

Bohn, M. et al. (2006) Genetic evaluation of root complexity in maize. Acta Agronomica Hungarica, 54, $291-303$.

Cairns, J. et al. (2009) Mapping quantitative trait loci associated with root growth in upland rice (Oryza sativa L.) exposed to soil water-deficit in fields with contrasting soil properties. Field Crops Research, 114, 108-118. 
Chamarthi, S.K. et al. (2011) Trait mapping and molecular breeding. In: A. Pratap and J. Kumar (eds) Biology and Breeding of Food Legumes. CABI International, Oxford shire, pp. 296-313.

Chen, J.Y. et al. (2009) Identification of QTLs for phosphorus utilization efficiency in maize (Zea mays L.) across P levels. Euphytica, 167, 245-252.

Chen, Y.L. et al. (2011) Development of a novel semi-hydroponic phenotyping systemfor studying root architecture. Functional Plant Biology, 38, 355-363.

Cichy, K.A. et al. (2009a) QTL analysis of root architecture traits and low phosphorus tolerance in an Andean bean population. Crop Science, 49, 59-68.

Cichy et al. (2009b). QTL analysis of seed iron, zinc, and phosphorus levels in an Andean bean population. Crop Science, 49, 1742-1750.

Coudert, Y. et al. (2010) Genetic control of root development in rice, the model cereal. Trends in Plant Science, 15, $219-226$.

Courtois, B. et al. (2003) Locating QTLs controlling constitutive root traits in the rice population IAC165 $\times$ Co39. Euphytica, $134,335-345$.

Courtois, B. et al. (2009) Rice root genetic architecture: meta-analysis from a drought QTL database. Rice, 2, $115-128$.

de Dorlodot, S. et al. (2007) Root system architecture: opportunities and constraints for genetic improvement of crops. Trends in Plant Science, 12, 474-481.

Eathington, S.R. et al. (2007) Molecular markers in a commercial breeding program. Crop Science, 47, S154-S163.

Gai, J. et al. (2007) Identification, inheritance and QTL mapping of root traits related to tolerance to rhizospheric stresses in soybean (G. max (L.) Merr.). Frontiers of Agriculture in China, 1, 119-128.

Giuliani, M.M. et al. (2000) Comparative QTL analysis in maize for vertical root pulling resistance in the field and root traits in hydroponics. International Conference on Plant \& Animal Genome VIII, January 9-12, San Diego, California, p. 114.

Gowda, V.R.P. et al. (2011) Root biology and genetic improvement for drought avoidance in rice. Field Crop Research 122, $1-13$.

Gregory, P.J. et al. (2009) Root phenomics of crops: opportunities and challenges. Functional Plant Biology, 36, 922-929.

Guingo, E. et al. (1998) Genetic analysis of root traits in maize. Agronomie, 18, 225-235.

Gupta, P.K. et al. (2010) Marker assisted selection as a component of conventional plant breeding. Plant Breeding Reviews, 33, $145-217$.

Hemamalini, G.S. et al. (2000) Molecular marker assisted tagging of morphological and physiological traits under two contrasting moisture regimes at peak vegetative stage in rice (Oryza sativa L.). Euphytica, 112, 69-76.

Hochholdinger, F. and Tuberosa, R. (2009) Genetic and genomic dissection of maize root development and architecture. Current Opinion in Plant Biology, 12, 172-177.

Horii, H. et al. (2006) Quantitative trait loci for adventitious and lateral roots in rice. Plant Breeding, 125, 198-200.

Hund, A. et al. (2009) Growth of axile and lateral roots of maize: I. Development of a phenotyping platform. Plant Soil, 325, 335-349.

Hund, A. et al. (2004) QTL controlling root and shoot traits of maize seedlings under cold stress. Theoretical and Applied Genetics, $109,618-629$.

Ikeda, H. et al. (2007) Genetic analysis of rooting ability of transplanted rice (Oryza sativa L.) under different water conditions. Journal of Experimental Botany, 58, 309-318.

Jackson, S.A. et al. (2011) Sequencing crop genomes: approaches and applications. The New Phytologist, 191, 915-925.

Kalliokoski, T. et al. (2008) Coarse root architecture of three boreal tree species growing in mixed stands. Silva Fenn, 42, 189-210.

Kamoshita, A. et al. (2002a) Mapping QTL for root morphology of a rice population adapted to rainfed lowland conditions. Theoretical and Applied Genetics, 104, 880-893.

Kamoshita, A. et al. (2002b) Effects of phenotyping environment on identification of quantitative trait loci for rice root morphology under anaerobic conditions. Crop Science, 42, 255-265.

Kashiwagi, J. et al. (2005) Genetic variability of drought-avoidance root traits in the mini-core germplasm collection of chickpea (Cicer arietinum L.). Euphytica, 146, 213-222.

Kashiwagi, J. et al. (2006) Variability of root length density and its contributions to seed yield in chickpea (Cicer arietinum L) under terminal drought stress. Field Crop Research, 95, 171-181.

Kell, D.B. (2011) Breeding crop plants with deep roots: their role in sustainable carbon, nutrient and water sequestration. Annals of Botany, 108, 407-418.

Kholová, J. et al. (2010b) Constitutive water conserving mechanisms are correlated with the terminal drought tolerance of pearl millet [Pennisetum glaucum (L.) R. Br.]. Journal of Experimental Botany, 61, 369-377.

Kholová, J. et al. (2010a) Terminal drought-tolerant pearl millet [Pennisetum glaucum (L.) R. Br.] have high leaf ABA and limit transpiration at high vapour pressure deficit. Journal of Experimental Botany, 61, 1431-1440.

Khowaja, F.S. et al. (2009) Improved resolution in the position of drought-related QTLs in a single mapping population of rice by meta-analysis. BMC Genomics, 10, 276. 
Kulwal, P.L. et al. (2011) Genomics interventions in crop breeding for sustainable agriculture. In: Encyclopedia of Sustainability Science and Technology. Springer, New York, DOI 10.1007/978-1-4419-0851-3.

Lambers, H. et al. (2008) Plant Physiological Ecology, Second edition. Springer, New York.

Landi, P. et al. (2007) Root-ABA1 QTL affects root lodging, grain yield, and other agronomic traits in maize grown under well-watered and water-stressed conditions. Journal of Experimental Botany, 58, 319-326.

Landi, P. et al. (2002) Detection of QTLs for vertical root pulling resistance in maize and overlap with QTLs for root traits in hydroponics and for grain yield under different water regimes. Maydica, 47, 233-243.

Landi, P. et al. (2010) Characterization of root-yield-1.06, a major constitutive QTL for root and agronomic traits in maize across water regimes. Journal of Experimental Botany, 61, 3553-3562.

Laperche, A. et al. (2006) A simplified conceptual model of carbon/nitrogen functioning for QTL analysis of winter wheat adaptation to nitrogen deficiency. Theoretical and Applied Genetics, 113, 1131-1146.

Lebreton, C. et al. (1995) Identification of QTL for drought responses in maize and their use in testing causal relationships between traits. Journal of Experimental Botany, 46, 853-865.

Levitt, J. (1972) Responses of Plants to Environmental Stresses, Academic Press, New York.

Li, Y.D. et al. (2005b) QTL mapping of phosphorus deficiency tolerance in soybean (Glycine max L. Merr.). Euphytica, 142, $137-142$.

Li, Z. et al. (2005a) QTL mapping of root traits in a doubled haploid population from a cross between upland and lowland japonica rice in three environments. Theoretical and Applied Genetics, 110, 1244-1252.

Li, J.Z. et al. (2009). Root and shoot traits responses to phosphorus deficiency and QTL analysis at seedling stage using introgression lines of rice. Journal of Genetics and Genomics, 36, 173-183.

Li, Z.K. et al. (2010) Analysis of QTLs for root traits at seedling stage using an immortalized $\mathrm{F}_{2}$ population of wheat. Acta Agronomica Sinica, 36, 442-448.

Li, Z.X. et al. (2007) Molecular mapping of QTLs for root response to phosphorus deficiency at seedling stage in wheat (Triticum aestivum L.). Proceedings of the National Academy of Sciences of the United States of America, 17, 1177-1184.

Liang, Q. et al. (2010) QTL analysis of root traits as related to phosphorus efficiency in soybean. Annals of Botany, 106, 223234.

Liao, H. et al. (2004) Genetic mapping of basal root gravitropism and phosphorus acquisition efficiency in common bean. Functional Plant Biology, 31, 959-970.

Liu, J. et al. (2008) Mapping QTLs for root traits under different nitrate levels at the seedling stage in maize (Zea mays L.). Plant Soil, 305, 253-265.

Ma, H.X. et al. (2005) Molecular mapping of a quantitative trait locus for aluminium tolerance in wheat cultivar Atlas 66. Theoretical and Applied Genetics, 112, 51-57.

Mace, E.S. et al. (2012) QTL for nodal root angle in sorghum (Sorghum bicolor L. Moench) co-locate with QTL for traits associated with drought adaptation. Theoretical and Applied Genetics, 12, 97-109.

MacMillan, K. et al. (2006) Assessing the importance of genotype $\times$ environment interaction for root traits in rice using a mapping population. I: a soil-filled box screen. Theoretical and Applied Genetics, 113, 977-986.

Mano, Y. et al. (2005) QTL mapping of adventitious formation under flooding conditions in tropical maize (Zea mays L.) seedlings. Breeding Science, 55, 343-347.

Manschadi, A.M. et al. (2008) Genotypic variation in seedling root architectural traits and implications for drought adaptation in wheat (Triticum aestivum L.). Plant Soil, 303, 115-129.

Miguel, M.A. et al. (2009) QTL mapping of basal root whorl number in common bean (Phaseolus vulgaris L). ASACSSA-SSAA International Annual Meeting, November 1-5, 2009, Pittsburg, PA. http://a-c-s.confex.com/crops/2009am/ webprogram/Paper55496.html.

Mir, R.R. et al. (in press) Evolving molecular marker technologies for plant genetics research and breeding applications. In: T. Lübberstedt and R.K. Varshney (eds) Diagnostics in Plant Breeding. Springer, The Netherlands.

Morrell, P.L. et al. (2012) Crop genomics: advances and applications. Nature Reviews Genetics, 13, 85-96.

Myles, S. et al. (2009) Association mapping: critical considerations shift from genotyping to experimental design. Plant Cell, 21, 2194-2204.

Nagel, K.A. et al. (2009) Temperature responses of roots: impact on growth, root system architecture and implications for phenotyping. Functional Plant Biology, 36, 947-959.

Norton, G.J. and Price, A.H. (2009) Mapping of quantitative trait loci for seminal root morphology and gravitropic response in rice. Euphytica, 166, 229-237.

Obara, M. et al. (2010) Fine-mapping of qRL6.1, a major QTL for root length of rice seedlings grown under a wide range of $\mathrm{NH}_{4}{ }^{+}$ concentrations in hydroponic conditions. Theoretical and Applied Genetics, 121, 535-547.

Ochoa, I.E. et al. (2006) QTL analysis of adventitious root formation in common bean under contrasting phosphorus availability. Crop Science, 46, 1609-1621. 
Omori, F. and Mano, Y. (2007) QTL mapping of root angle in $\mathrm{F}_{2}$ populations from maize "B73" $\times$ teosinte "Zea luxurians." Plant Root, 1, 57-65.

Ouertani, K. et al. (2010) Quantitative trait loci (QTL) that underlie root and shoot traits in the soybean [Glycine max (L.) Merr.] "Hartwig" by "Flyer" recombinant inbred line (RIL) population. Internation Conference on Plant \& Animal Genomes XVIII, January 9-13, 2010, Town and Country Convention Center, San Diego, California. http://www.intlpag.org/18/abstracts/P05f_PAGXVIII_395.html.

Paterson, A.H. et al. (2009) The Sorghum bicolor genome and the diversification of grasses. Nature, 457, 551-556.

Petrarulo, M. et al. (2009) Mapping QTLS for root morphological traits in durum wheat. International Symposium on Root Research and Applications, 2-4 September, Boku, Vienna, Austria. http://asrr.boku.ac.at/fileadmin/files/ RRcd/session07/poster/195.pdf

Priya, P.S. et al. (2009) Genome-wide analyses of rice root development QTLs and development of an online resource, Root browse. Bioinformation, 3, 279-281.

Qu, Y. et al. (2008) Mapping QTLs of root morphological traits at different growth stages in rice. Genetica, 133, 187-200.

Rafalski, A. (2010) Association genetics in crop improvement. Current Opinion in Plant Biology, 13, $174-180$.

Ren, Y. et al. (2011) Major quantitative trait loci for seminal root morphology of wheat seedlings. Molecular Breeding, 30, $139-148$.

Reynolds, M. and Tuberosa, R. (2008) Translational research impacting on crop productivity in drought prone environments. Current Opinion in Plant Biology, 11, 171-179.

Ribaut, J.M. and Ragot, M. (2007) Marker-assisted selection to improve drought adaptation in maize: the backcross approach, perspectives, limitations, and alternatives. Journal of Experimental Botany, 58, 351-360.

Ruta, N. et al. (2010) QTLs for the elongation of axile and lateral roots of maize in response to low water potential. Theoretical and Applied Genetics, 120, 621-631.

Salvi, S. and Tuberosa, R. (2005) To clone or not to clone plant QTLs: present and future challenges. Trends in Plant Science, 10, 297-304.

Salvi, S. and Tuberosa, R. (2007) Cloning QTLs in plants. In: R.K. Varshney and R. Tuberosa (eds) Genomics-Assisted Crop Improvement, Vol. I. Springer, New York, pp. 207-226.

Sanguineti, M.C. et al. (2007) Genetic dissection of seminal root architecture in elite durum wheat germplasm. Annals of Applied Biology, 151, 291-305.

Schmutz, J. et al. (2010) Genome sequence of the palaeopolyploid soybean. Nature, 463, 178-183.

Schnable, P.S. et al. (2009) The B73 maize genome: complexity, diversity, and dynamics. Science, 326, $1112-1115$.

Sharma, S. et al. (2011) Dissection of QTL effects for root traits using a chromosome arm-specific mapping population in bread wheat. Theoretical and Applied Genetics, 122, 759-769.

Shen, L. et al. (2001) Evaluation of near-isogenic lines of rice introgressed with QTLs for root depth through marker-aided selection. Theoretical and Applied Genetics, 103, 427-437.

Sheshshayee, M.S. et al. (2011) Phenotyping for root traits and their improvement through biotechnological approaches to sustaining crop productivity. In: A.C. de Oliveira and R.K. Varshney (eds) Root Genomics. Springer-Verlag, Berlin Heidelberg, pp. 205232.

Smita, S. et al. (2011) QlicRice: a web interface for abiotic stress responsive QTL and loci interaction channels in rice. Database, Doi: 10.1093/database/bar037.

Srividya, A. et al. (2010). Identification and mapping of landrace derived QTL associated with yield and its components in rice under different nitrogen levels and environments. International Journal of Plant Breeding and Genetics, 4, 210227.

Steele, K.A. et al. (2006) Marker-assisted selection to introgress rice QTLs controlling root traits into an Indian upland rice variety. Theoretical and Applied Genetics, 112, 208-221.

Steele, K.A. et al. (2007) Field evaluation of upland rice lines selected for QTLs controlling root traits. Field Crop Research, 101, $180-186$.

Swamy, M.B.P. et al. (2011) Meta-analysis of grain yield QTL identified during agricultural drought in grasses showed consensus. BMC Genomics, 12, 319.

Tuberosa, R. and Salvi, S. (2006) Genomics approaches to improve drought tolerance in crops. Trends in Plant Science, 11, 405-412.

Tuberosa, R. and Salvi, S. (2007) From QTLs to genes controlling root traits in maize. In: J.H.J. Spiertz, P.C. Struik, and H.H. van Laar (eds) Scale and Complexity in Plant Systems Research: Gene Plant Crop Relations. Springer, Dordrecht, The Netherlands, pp. 15-24.

Tuberosa, R. et al. (2002a) Identification of QTLs for root characteristics in maize grown in hydroponics and analysis of their overlap with QTLs for grain yield in the field at two water regimes. Plant Molecular Biology, 48, 697-712.

Tuberosa, R. et al. (2003) Searching for quantitative trait loci controlling root traits in maize: a critical appraisal. Plant Soil, 255, $35-54$. 
Tuberosa, R. et al. (2002b) Mapping QTLs regulating morpho-physiological traits and yield: case studies, short comings and perspectives in drought-stressed maize. Annals of Botany, 89, 941-963.

Tuberosa, R. et al. (2011) Genomics of root architecture and functions in maize. In: A. Costa de Oliveira and R.K. Varshney (eds) Root Genomics. Springer, Berlin Heidelberg, pp. 179-204.

Uga, Y. et al. (2008) QTL underlying natural variation in stele and xylem structures of rice root. Breeding Science, 58, 7-14.

Uga, Y. et al. (2011) Dro1, a major QTL involved in deep rooting of rice under upland field conditions. Journal of Experimental Botany, 62, 2485-2494.

Ulaganathan, K. et al. (2007) Physical integration of root QTLs with the japonica genome and prediction of probable candidate genes for drought tolerance in rice. Molecular Plant Breeding, 5, 213-214.

Varshney, R.K. et al. (2005) Genomics-assisted breeding for crop improvement. Trends in Plant Science, 10, 621-630.

Varshney, R.K. et al. (2006) Advances in cereal genomics and applications in crop breeding. Trends in Biotechnology, 24, 490-499.

Varshney, R.K. et al. (2011a) Agricultural biotechnology for crop improvement in a variable climate: hope or hype? Trends in Plant Science, 16, 363-371.

Varshney, R.K. et al. (2011b) Genomics and physiological approaches for root trait breeding to improve drought tolerance in chickpea (Cicer arietinum L.). In: A.D. de Oliveira and R.K. Varshney (eds) Root Genomics. Springer, Berlin Heidelberg, pp. 233-250.

Varshney, R.K. et al. (2010) Legume genomics and breeding. Plant Breeding Reviews, 33, 257-304.

Varshney, R.K. et al. (2009) Next-generation sequencing technologies and their implications for crop genetics and breeding. Trends in Biotechnology, 27, 522-530.

Varshney, R.K. et al. (2012) Draft genome sequence of pigeonpea (Cajanus cajan), an orphan legume crop of resource-poor farmers. Nature Biotechnology, 30, 83-89.

Venuprasad, R. et al. (2002) Tagging quantitative trait loci associated with grain yield and root morphological traits in rice (Oryza sativa L.) under contrasting moisture regimes. Euphytica, 128, 293-300.

Washington, E. et al. (2009) A genetic analysis of root traits in soybean plants grown in the greenhouse and in the field. International Conference on Plant \& Animal Genomes XVII, January 10-14, 2009, Town \& Country Convention Center, San Diego, California. http://www.intl-pag.org/17/abstracts/P05f_PAGXVII_374.html.

Williams, B. et al. (2012) Genetic analysis of root and shoot traits in the "Essex" by "Forrest" recombinant inbred line (RIL) population of soybean [Glycine max (L.) Merr.]. Journal of Plant Genome Sciences, 1, 1-9.

Xu, J.C. et al. (2001) QTL mapping of the root traits in rice seedling. Acta Genet Sin, 28, 433-438.

Yan, X. et al. (2004) QTL mapping of root hair and acid exudation traits and their relationship to phosphorus uptake in common bean. Plant Soil, 265, 17-29.

Yazdanbakhsh, N. and Fisahn, J. (2009) High throughput phenotyping of root growth dynamics, lateral root formation, root architecture and root hair development enabled by PlaRoM. Functional Plant Biology, 36, 938-946.

Yue, B. et al. (2008) Identification of quantitative trait loci for four morphologic traits under water stress in rice (Oryza sativa L.). Journal of Genetics and Genomics 35, 569-575.

Yue, B. et al. (2006) Genetic basis of drought resistance at reproductive stage in rice: separation of drought tolerance from drought avoidance. Genetics, 172, 1213-1228.

Zaman-Allah, M. et al. (2011) A conservative pattern of water use, rather than deep or profuse rooting, is critical for the terminal drought tolerance of chickpea. Journal of Experimental Botany, 62, 4239-4252.

Zhang, D. et al. (2009) Detection of quantitative trait loci for phosphorus deficiency tolerance at soybean seedling stage. Euphytica, $167,313-322$.

Zhang, J. et al. (2001) Locating genomic regions associated with components of drought resistance in rice: comparative mapping within and across species. Theoretical and Applied Genetics, 103, 19-29.

Zheng, B.S. et al. (2003) Mapping QTLs and candidate genes for rice root traits under different water-supply conditions and comparative analysis across three populations. Theoretical and Applied Genetics, 107, 1505-1515.

Zhu, J.M. et al. (2005a) Mapping of QTL controlling root hair length in maize (Zea mays L.) under phosphorus deficiency. Plant Soil, 270, 299-310.

Zhu, J.M. et al. (2005b) Mapping of QTLs for lateral root branching and length in maize (Zea mays L.) under differential phosphorus supply. Theoretical and Applied Genetics, 111, 688-695.

Zhu, J.M. et al. (2006) Detection of quantitative trait loci for seminal root traits in maize (Zea mays L.) seedlings grown under differential phosphorus levels. Theoretical and Applied Genetics, 113, 1-10.

Zobel, R.W. and Weisel, Y. (2010) A plant root system architectural taxonomy: a framework for root nomenclature. Plant Biosystems, 144, 507-512. 